\title{
A SPARSITY-DRIVEN JOINT IMAGE REGISTRATION AND CHANGE DETECTION TECHNIQUE FOR SAR IMAGERY
}

\author{
Lam H. Nguyen ${ }^{1}$ and Trac D. Tran ${ }^{2}$ \\ ${ }^{1}$ U.S. Army Research Laboratory \\ Adelphi, MD 20783 USA \\ ${ }^{2}$ The Johns Hopkins University \\ Baltimore, MD 21218 USA
}

\begin{abstract}
This paper presents a novel Sparsity-driven joint Image REgistration and Change Detection (SIRE-CD) technique for SAR imagery. The proposed algorithm simultaneously performs two main tasks: (i) locally register the test and reference images; and (ii) perform the change detection between the two. The key innovative concept here is the sparsity-driven transformation of the signatures from the reference image to match to those of the test image at the local image patch level. In other words, we are constructing a large dictionary from the reference data and use that to find the sparsest representation that best approximates the new incoming test data. The accuracy level of the approximation determines the detected changes between the reference and the test image. We demonstrate the performance of this technique using both simulated data and real SAR imagery from the Army Research Laboratory ultra-wideband (UWB) SAR forward-looking radar.
\end{abstract}

Index Terms - image registration, change detection, synthetic aperture radar (SAR), sparse representation, compressed sensing.

\section{INTRODUCTION}

Change detection is a popular and crucial image processing process in numerous applications such as medical imaging, agriculture, and other remote sensing tasks. In synthetic aperture radar (SAR) application, many different change detection techniques have been developed to detect targets from changes in SAR images, ranging from difference test, ratio test, to subspace projection [1]-[4]. These SAR images from the same area are collected and generated at different times (minutes, hours, or even days). Any changes between the SAR images that are detected by the algorithm are considered to be targets of interest that should be further investigated.

There are two major challenges that any change detection algorithm must face. The first challenge is the image registration problem. The two SAR images - referred to as the reference image and the test image in a change detection algorithm - usually are not aligned since in general it is not possible to keep the radar platform in the exact geometry (radar aperture) from one run to another. This results in a translation and even some slight rotation between the two. Performances of change detection often depend on (besides the techniques involved) how well image registration can be achieved. If the alignment was not well performed prior to the application of the change detection algorithm, the resulting change detection performance will degrade significantly as a function of the accuracy of the alignment. In addition, the degree of misalignment between the two images usually varies throughout large areas. Thus, it might not be possible to achieve a good registration between the two images (test and reference) globally. We hypothesize that local geometry changes can be best captured by performing image registration locally.

The second challenge is the suppression of the signatures from common objects that appear in both test and reference image scenes. In the ideal situation, after the two images are carefully registered, the signatures of the objects that are presented in both passes would be almost the same in both images. Hence, the signatures from common objects that appear in both image scenes should be well suppressed by the change detection algorithm. However, in reality, the image signatures of the same object are often different in the two images due to many reasons (radar calibration problem, slight change in aspect angle, noise, etc.). In the resulting change detection image, these different signatures of a common object would result in large differences, which would result in a high false-alarm rate. Current state-of-theart change detection algorithms often have difficulty in suppressing these differences since they simply detect any anomalies between the signatures of these common objects.

Our proposed algorithm simultaneously addresses both problems of registration and suppression of common objects via a series of local sparse optimization processes. We construct a redundant dictionary from the reference data and use that dictionary to approximately represent the new incoming test data. The concept of sparsity in our approach contains a principal component analysis (PDA) flavor - only the most influential signatures are allowed to contribute to the linear approximation. However, unlike classic PDA approaches, ours is completely deterministic: the algorithm operates directly on the collected data and we have not made any effort in training the dictionary either. Finally, our subspace approach is locally adaptive: at the image patch level, we search for the optimal subspace that can be constructed from the reference data via a sparsity-guidance optimization process. This search feature accomplishes both critical tasks of registration and change detection simultaneously.

\section{SPARSITY-DRIVEN JOINT IMAGE REGISTRATION AND CHANGE DETECTION}

\subsection{Notation}

Bold-faced lowercase characters denote vectors while bold-faced uppercase characters denote matrices. The lowercase subscript $i$ and $j$ used throughout the paper is reserved to index either image pixel as in $x_{i j}$ or image patch location $\boldsymbol{X}_{i j}$. The $L_{p}$-norm of the vector $\boldsymbol{x}$ is defined as

$$
\|\mathbf{x}\|_{p}=\sqrt[p]{\sum_{i}\left|x_{i}\right|^{p}} .
$$


whereas the $L_{0}$-norm of $\boldsymbol{x}$ is simply its number of significant (nonzero) elements.

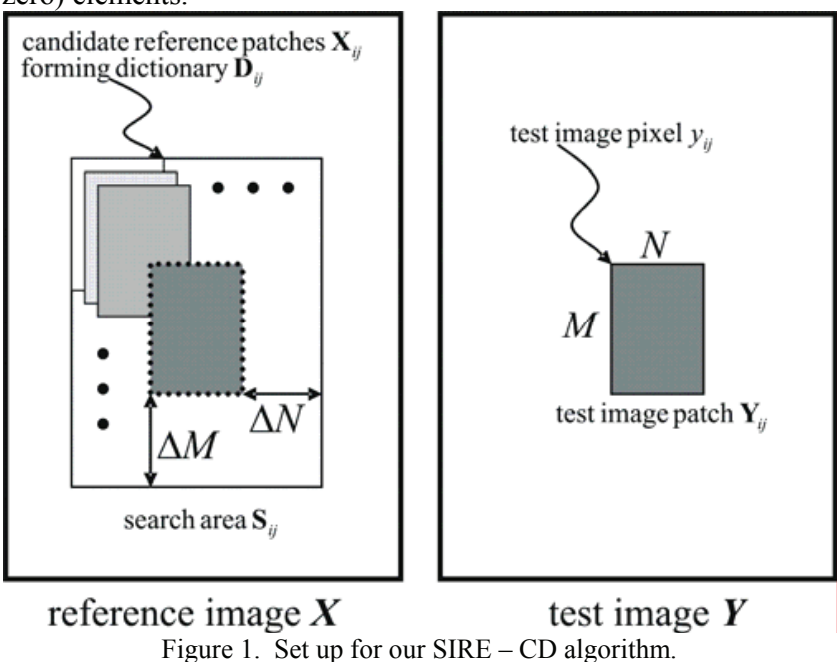

\subsection{Compressed Sensing (CS) Framework}

Supposing $\mathbf{x}$ is a length- $N$ signal, it is said to be $K$-sparse (or $K$ compressible) if $\mathrm{x}$ can be well approximated using $K<<N$ coefficients under some linear transform (e.g. DWT, DFT, or some constructed dictionary). Such a signal can be acquired through a series of incoherent linear projections

$$
\mathbf{y}=\Phi \mathbf{x}
$$

where $\mathrm{y}$ is the vector with $M$ entries containing the measurements and $\Phi$ represents an $M \times N$ incoherent sensing matrix. The CS framework asserts that $\boldsymbol{x}$ can be faithfully recovered from only $M=$ $O(K \log N)$ measurements via solving the following sparsitydriven problem

$$
\hat{\boldsymbol{\alpha}}=\arg \min \|\boldsymbol{\alpha}\|_{0} \text { s.t. } \mathbf{y}=\boldsymbol{\Phi} \mathbf{x}=\boldsymbol{\Phi} \boldsymbol{\Psi} \boldsymbol{\alpha}=\mathbf{A} \boldsymbol{\alpha}
$$

and the original signal can be reconstructed as $\mathbf{x}^{*}=\Psi \alpha^{*}$. Under some mild conditions, the $L_{0}$-norm minimization problem above can be efficiently solved by recasting it as a convex linear programming problem [5]

$$
\hat{\boldsymbol{\alpha}}=\arg \min \|\boldsymbol{\alpha}\|_{1} \text { s.t. } \quad \mathbf{y}=\boldsymbol{\Phi} \mathbf{x}=\boldsymbol{\Phi} \boldsymbol{\Psi} \boldsymbol{\alpha}=\mathbf{A} \boldsymbol{\alpha}
$$

In any case, the reconstructed signal can be roughly regarded as the sparsest (or most compressible) solution from many candidate solutions of the under-determined equation in (1).

Although our work is certainly inspired by CS, there are quite a few significant differences: (i) we do not need CS measurements via random projections - our SAR data is already collected; (ii) the sparsifying transform $\boldsymbol{\Psi}$ is constructed from data patches; and (iii) most importantly, our algorithm operates at local image patch level - the sparsity $K$ changes from patch to patch. In fact, behavior of $K$ allows us to perform effective change detection.

\subsection{Sparse Representation via Dictionary of Reference Data}

Consider the two SAR images $\mathbf{X}$ and $\mathbf{Y}$ (as depicted in Figure 1) which cover the same physical area but are collected at two different times. We would like to first register the two images $\mathbf{X}$ and $\mathbf{Y}$, and then identify any changes between them. The test image $\boldsymbol{Y}$ is divided into many overlapping image patches $\boldsymbol{Y}_{i j}$, each represented as an $M x N$ matrix. Define $\boldsymbol{y}_{i j}$ as an $M N \times 1$ column vector constructed from stacking $N$ columns of $\boldsymbol{Y}_{i j}$, i.e.,

$$
\boldsymbol{y}_{i j}=\left[\boldsymbol{y}_{: j}^{\mathrm{T}} \boldsymbol{y}_{:, j+1}^{\mathrm{T}} \ldots \boldsymbol{y}_{: j+N-1}^{\mathrm{T}}\right]^{\mathrm{T}}
$$

where

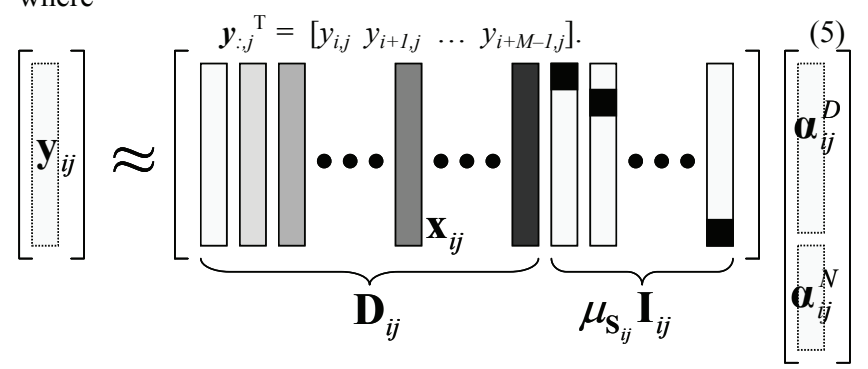

Figure 2. Sparse representation of test image patch $\boldsymbol{y}_{i j}$ via a dictionary of previously collected reference data $\boldsymbol{x}_{i j}$.

Next, define the corresponding $(M+2 \Delta M) \times(N+2 \Delta N)$ search area $\boldsymbol{S}_{i j}$ in the reference image $\boldsymbol{X}$ as follows

$$
\mathbf{S}_{i j} \equiv\left[\begin{array}{ccc}
x_{i-\Delta M, j-\Delta N} & \cdots & x_{i-\Delta M, j+\Delta N+N-1} \\
\vdots & \ddots & \vdots \\
x_{i+\Delta M+M-1, j-\Delta N} & \cdots & x_{i+\Delta M+M-1, j+\Delta N+N-1}
\end{array}\right] .
$$

Obviously, the size of the search area controls the robustness of the algorithm with respect to registration. With this set-up, SIRE-CD can effectively handle any local registration mismatch within the range $[ \pm \Delta M, \pm \Delta N]$. From the above search area $\boldsymbol{S}_{i j}$, we construct the dictionary $\boldsymbol{D}_{i j}$ which consists of $(2 \Delta M+1)(2 \Delta N+1)$ atoms, each of exactly the same dimension as the test vector $\boldsymbol{y}_{i j}$ as follows

$\boldsymbol{D}_{i j}=\left[\begin{array}{llllll}\boldsymbol{x}_{i-\Delta M, j-\Delta N} & \boldsymbol{x}_{i-\Delta M, j-\Delta N+1} & \ldots & \boldsymbol{x}_{i-\Delta M+1, j-\Delta N} & \ldots & \boldsymbol{x}_{i+\Delta M, j+\Delta N}\end{array}\right]$

where each $M N \times 1$ atom $\boldsymbol{x}_{i j}$ is constructed in similar fashion as in (1) and (2) but from the reference data $\boldsymbol{X}$. The dictionary $\boldsymbol{D}_{i j}$ contains all possible reference image patches $\boldsymbol{X}_{i j}$ (in vectorized format $\boldsymbol{x}_{i j}$ ) that fit within the search area $\boldsymbol{S}_{i j}$ as described in (4)-(5).

\subsection{Sparse Optimization}

For every given test image patch $\boldsymbol{x}_{i j}$, we attempt to solve the following inverse problem for the sparsest coefficient vector $\boldsymbol{\alpha}_{i j}$

$$
\mathbf{y}_{i j}=\left[\begin{array}{ll}
\mathbf{D}_{i j} & \mu_{\mathbf{S}_{i j}} \mathbf{I}_{i j}
\end{array}\right]\left[\begin{array}{c}
\boldsymbol{\alpha}_{i j}^{D} \\
\boldsymbol{\alpha}_{i j}^{N}
\end{array}\right] \equiv \mathbf{A}_{i j} \boldsymbol{\alpha}_{i j}
$$

where $\mu_{S_{i j}}$ is the mean of the reference search area $\boldsymbol{S}_{i j}$ in (6) and $\boldsymbol{I}_{i j}$ is the size- $M N$ identity matrix that is employed to account for noises and any real changes [6]. In other words, we solve the following problem

$$
\hat{\boldsymbol{\alpha}}_{i j}=\arg \min \left\|\boldsymbol{\alpha}_{i j}\right\|_{0} \text { s.t. } \quad \mathbf{y}_{i j}=\mathbf{A}_{i j} \boldsymbol{\alpha}_{i j}
$$

and find the best sparse approximation of the test image patch $\boldsymbol{y}_{i j}$ as a linear combination of the corresponding local reference image patches stored in the dictionary $\boldsymbol{D}_{i j}$

$$
\hat{\mathbf{y}}_{i j}=\mathbf{D}_{i j} \hat{\boldsymbol{\alpha}}_{i j}^{D} .
$$

There are numerous techniques for solving (9) for the sparsest solution available in the compressed sensing community: linear programming, orthogonal matching pursuit, gradient projection, subspace pursuit, sparsity adaptive matching pursuit, etc. [5]-[8]. In this paper, we employed subspace pursuit [8] with the input sparsity $\mathrm{K}$ set to $2-5 \%$ the image patch dimension.

\subsection{Change Detection}

Define several measures of prediction error as follows

$$
\mathbf{e}_{i j} \equiv\left|\mathbf{y}_{i j}-\hat{\mathbf{y}}_{i j}\right|, \quad \mathbf{e}_{i j}^{\prime} \equiv\left\|\hat{\boldsymbol{\alpha}}_{i j}\right\|_{1} \mathbf{e}_{i j}, \quad \mathbf{e}_{i j}^{\prime \prime} \equiv\left\|\hat{\boldsymbol{\alpha}}_{i j}\right\|_{1} \mathbf{e}_{i j} / \sigma_{\mathbf{S}_{i j}}
$$


where $\sigma_{S_{i j}}^{2}$ is the variance of the reference search area $\boldsymbol{S}_{i j}$. The accuracy of the sparse representation above and the degree of sparsity roughly measured as the $L_{l}$-norm of the optimal coefficient vector $\left\|\hat{\boldsymbol{\alpha}}_{i j}\right\|_{1}$ are critical information for us to determine if changes have taken place within the image area near location $i j$ or not.

Let us consider the first hypothesis where there is no change in the image scene. In the ideal noiseless case, if the signatures from the test image and the reference image are identical, the solution $\hat{\boldsymbol{\alpha}}_{i j}$ vector would include only one nonzero element of unity magnitude. In reality, there are always subtle changes between the signatures from the two images. Thus, the solution $\hat{\boldsymbol{\alpha}}_{i j}$ would include a few nonzero small-magnitude elements to compensate for the differences and its $L_{l}$-norm is insignificant. On the other hand, when there are changes within the local image scene, the test image patch cannot be well represented by the linear combination of reference atoms in the dictionary (since we impose the restriction that sparsity $K$ is very low compared to the dimension of the test image patch). This yields a noisy prediction and the resulting differences would indicate the changes. Here, the resulting $L_{l}$-norm of the best solution $\left\|\hat{\boldsymbol{\alpha}}_{i j}\right\|_{1}$ would be significantly higher, indicating a detected change.

2.6. Algorithm Summary and Parameter Settings In short, the overall proposed CD algorithm operates as follows

- Inputs: reference image $\boldsymbol{X}$ and test image $\boldsymbol{Y}$

- Parameter settings:

- Image patch size: $M \times N=1 \times 1$ to $2 \times 2 m$

Targeted sparsity level: $K=0.02 M N$ to $K=0.05 M N$

Search window size: $\Delta M=M, \Delta N=N$

$50 \%$ overlap between image patches: $\delta i=M / 2 ; \delta j=N / 2$

- Partition test image $\boldsymbol{Y}$ into overlapping test image patches

- For each test image patch $\boldsymbol{Y}_{i j}$ Construct local dictionary $\boldsymbol{D}_{i j}$ from the reference as in (7) Solve the $L_{0}$-norm optimization problem in (8)-(9)

Compute the sparse prediction patch as in (10)

Compute the residual prediction errors from (11)

Increment $i$ and $j$ by $\delta i$ and $\delta j$ to cover the entire test image.

- Outputs: The global prediction $\hat{\boldsymbol{Y}}, L_{1}$-norm coefficient map $\boldsymbol{L}$, and scaled change detection $\hat{E}$ image are generated from averaging all local patches $\hat{\boldsymbol{y}}_{i j},\left\|\hat{\boldsymbol{\alpha}}_{i j}\right\|_{1}, \boldsymbol{e}_{i j}$, respectively, in overlap-add fashion.

\section{RESULTS}

3.1. Change detection results using SAR Simulated Data To validate the theory, we first test our SIRE-CD algorithm on simulated imagery. Figure 3 a shows a reference SAR image formed using SAR simulation data. There are eight clutter objects of different amplitudes. Figure $3 \mathrm{~b}$ depicts a test SAR image that is collected and generated at a different time. There are three new targets introduced in the scene. The first and second targets are located in the clear area. The third target is located right next to the first clutter object. Its amplitude is lower than its neighboring clutter object. All clutter signatures presented in both scenes are different between the test and the reference image. The differences in the signatures (shapes and amplitudes) represent the radar calibration mismatch between two different runs. In addition to the signature mismatches, there is also a misalignment between the two images due to variations in geometry (radar aperture) between the two runs. Figure $3 \mathrm{c}$ shows the standard difference change detection result using the test and reference images after applying a global image registration. The suppression of signatures for these common objects completely fails due to the local image registration problem and the changes in signatures between runs.

Figure 4 a shows the prediction image $\hat{\boldsymbol{Y}}$ using our SIRECD. The algorithm has successfully aligned and matched signatures of the seven common objects. On the other hand, the transformation of the signatures of the first common object together with a weaker target located next to it, and the signatures of the two targets in the clear are less successful. Figure $4 \mathrm{~b}$ shows the global difference image $\boldsymbol{E}$ whose pixels at three target locations show up clearly while signatures of the other four common objects are well suppressed. Figure 4c shows the false color plot of the $L_{1}$ coefficient map $\boldsymbol{L}$, which tabulates all locally-optimal $L_{1}$-norm of coefficient vectors $\left\|\hat{\boldsymbol{\alpha}}_{i j}\right\|_{1}$. The response is highest at the location of the largest target in the clear (toward lower right corner) where the optimization search fails to yield a closely matched result and the best that it can offer is the set of coefficients $\hat{\boldsymbol{\alpha}}_{i j}$ that are quite large in magnitude. This $L_{1}$ coefficient map clearly shows valuable information that can be used to weight the change detection image to enhance the locations with target changes. Figure $4 d$ shows the final change detection image using the weighted prediction error $\boldsymbol{e}_{i j}$ as in (11): three target locations are detected while the clutter objects are completely eliminated. We have achieved a significant level of change detection performance using the SIRE-CD technique, even in this challenging case. Note that the technique handles very well the local registration mismatch. The sparsitydriven optimization process adaptively decides what the best local alignment for each signature is.

\subsection{Change detection results using $S A R$ imagery from ARL UWB SAR forward looking radar.}

The Army Research Laboratory has recently developed a forwardlooking SAR system for the detection of the obscured targets [9]. Figures $5 \mathrm{a}$ and $5 \mathrm{~b}$ show the reference and test SAR images. There are seven ground-truth targets (labeled with circles). Some targets have very small signature amplitudes and are located next to large clutter objects, representing very challenging situations. Figure $5 \mathrm{c}$ shows the false color plot of the $L_{l}$-norm coefficient map $\boldsymbol{L}$, already disclosing valuable information for the detection of targets. All seven targets are detected with two "legitimate" false alarms, one of which is caused by the spurious signal from the radar hardware. Figure $5 \mathrm{~d}$ shows the residual change detection image scaled by the $L_{1}$-norm coefficients and the standard deviations of the local reference patches as described in Section 2.

\section{SUMMARY}

In summary, we have presented a locally-adaptive sparsity-driven joint registration and change detection approach: at the image patch level, we search for the optimal subspace that can be constructed from the reference data via a $L_{0^{-}}$or $L_{1}$-norm sparsityguidance optimization process. The accuracy level of this representation determines the detected changes between the reference and the test image. The perfect alignment between the reference and test images is achieved by the built-in registrationthe sparse optimization process automatically decides what the best local alignment for each signature is. Surprisingly, the $L_{1^{-}}$ 
norm information is very effective in detecting the changes (target detection) between the two images. Combining this $L_{l}$-norm information and the change detection result should provide significant performance in change detection approach. We have demonstrated the SIRE-CD performance using both simulated and real forward-looking SAR data from ARL radar. Detailed statistical analysis/comparison is currently conducted and will be published in the near future.

\section{REFERENCES}

[1] E. Rignot and J. V. Zyl, "Change detection techniques for ERS-1 SAR data," IEEE Trans. on Geoscience and Remote Sensing, vol. 31, no. 4, pp. 896-906, Jul. 1993.

[2] M. Carlotto, "Detection and analysis of change in remotely sensed imagery with application to wide area surveillance," IEEE Trans. on Image Processing, vol. 6, no. 1, pp. 189-202, Jan. 1997.

[3] K. I. Ranney and M. Soumekh, "Signal subspace change detection in averaged multilook SAR imagery," IEEE Trans. on Geo. and Remote Sensing, vol. 44, no. 1, pp. 201-213, Jan. 2006.
[4] K. I. Ranney and H. C. Khatri, "Modified difference change detector for small targets in SAR imagery," IEEE Trans. on Aerospace and Electronic Systems, vol. 44, no. 1, Jan. 2008.

[5] E. Candès and M. Wakin, "An introduction to compressive sampling," IEEE Signal Processing Magazine, vol. 25, no. 2, pp. 21-30, Mar. 2008.

[6] J. Wright, A. Yang, A. Ganesh, S. Shastry, and Y. Ma, "Robust face recognition via sparse representation," IEEE Trans. on PAMI, vol. 31, Feb. 2009.

[7] J. Tropp and A. Gilbert, "Signal recovery from random measurements via orthogonal matching pursuit," IEEE Trans. on Information Theory, vol. 53, no. 12, pp. 4655-4666, Dec. 2007.

[8] W. Dai and O. Milenkovic, "Subspace pursuit for compressive sensing: Closing the gap between performance and complexity," Preprint, 2008.

[9] Marc Ressler, Lam Nguyen, Francois Koenig, David Wong, and Greory Smith, "The Army Research Laboratory (ARL) Synchronous Impulse Reconstruction (SIRE) Forward-Looking Radar," Proceedings of SPIE, Unmanned Systems Technology IX, Vol. 6561, May 2007.


Figure 3. Simulation SAR data. a) Reference image; b) Test Image; c) Difference change detection applied to two images (with global image registration).
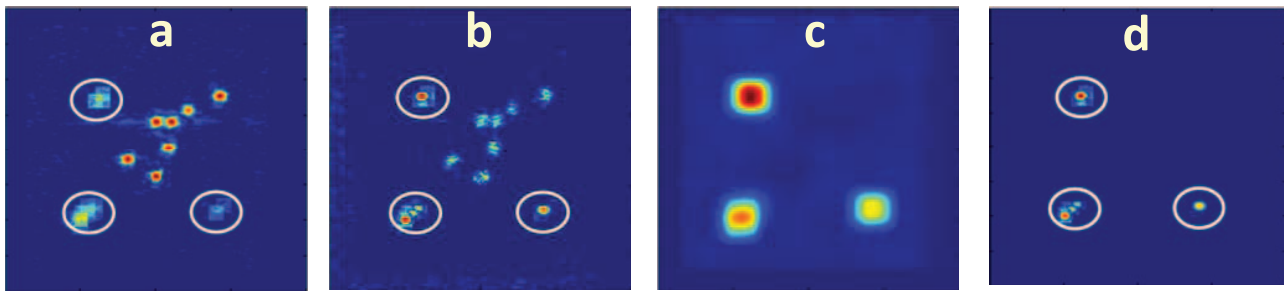

Figure 4. Change detection result. a) Sparse prediction image $\hat{\boldsymbol{Y}}$; b) Residual error image $\boldsymbol{E}$; c) L1-norm coefficient map $\boldsymbol{L}$; d) Scaled change detection image (residual image scaled by L1-norm) $\boldsymbol{E}$.


Figure 5. Real data from the Army Research Laboratory UWB forward-looking SAR. a) Reference image $\boldsymbol{X}$; b) Test image $\boldsymbol{Y}$; c) L1-norm of optimal coefficient map $\boldsymbol{L}$; d) Change detection image $\boldsymbol{E}$ (residual image $\boldsymbol{E}$ scaled by L1-norm and standard deviation of reference search area). 